\title{
Directional Structures Detection Based on Morphological Line-Segment and Orientation Functions
}

\author{
Iván R. Terol-Villalobos ${ }^{1}$, Luis A. Morales-Hernández ${ }^{2}$, \\ and Gilberto Herrera-Ruiz ${ }^{2}$ \\ ${ }^{1}$ CIDETEQ,S.C., Parque Tecnológico Querétaro S/N, SanFandila-Pedro Escobedo, \\ 76700, Querétaro Mexico \\ famter@ciateq. net.mx \\ ${ }^{2}$ Facultad en Ingeniería, Universidad Autónoma de Querétaro, 76000, México
}

\begin{abstract}
In the present paper a morphological approach for segmenting directional structures is proposed. This approach is based on the concept of the line-segment and orientation functions. The line-segment function is computed from the supremum of directional erosions. This function contains the sizes of the longest lines that can be included in the structure. To determine the directions of the line segments, the orientation function which contains the angles of the line segments it is built when the line-segment function is computed. Next, by combining both functions, a weighted partition is built using the watershed transformation. Finally, the elements of the partition are merged according to some directional and size criteria for computing the desired segmentation of the image using a RAG structure.
\end{abstract}

\section{Introduction}

Anisotropic structures are frequently found in many classes of images (materials, biometry images, biology, ...), however, few works dealing with directional analysis in morphological image processing have been carried out [123456 among others. It is maybe in the domain of fingerprint recognition, which is today the most widely used biometric features for personal identification, where the study of directional structures (called orientation-fields) based on directional structures detection is an active subject of research 789 . In fact, fingerprints can be considered as a structure composed by a set of line segments (see Fig. 1(a)). However, directional structure detection also plays a fundamental role in other domains 101112. Lee et al [10] propose a method based on oriented connectivity for segmenting solar loops, while Kass and Witkin 12 propose a method to analyze oriented patterns in wood grain. Given the interest in orientation pattern models for characterizing structures, this paper investigates the use of the mathematical morphology for modelling directional structures. As in the human vision, computer image processing of oriented image structures often requires a bank of directional filters or template masks, each of them sensitive to

D. Coeurjolly et al. (Eds.): DGCI 2008, LNCS 4992, pp. 458 469, 2008.

(C) Springer-Verlag Berlin Heidelberg 2008 
a specific range of orientations [1. Then, one investigates the use of an approach based on directional erosions. In the literature there exist several works to characterize directional structures based on the gradient computation that can be formalized in terms of mathematical morphology. See for example the works of 12139 . The problem of the gradients is that they work at pixel scale, they are very sensitives to noise and a final stage to enhance directional structures is required. Then, the main idea in this paper is focused on another approach that permits to take into account the whole context of the structures contained in the image. A local approach using the concept of line-segment function combined with the watershed transformation is used. In our case, the line-segment function is computed from the supremum of directional erosions. This function contains the information of the longest line segments that can be placed inside the structure. In order to know their orientations, a second image is defined by observing the construction of the line-segment function and its evolution. This second image is computed by detecting the orientation of the supremum of directional erosions. These local descriptors, for the element size and orientation, enable the identification of the directional structures based on the watershed transformation. This paper is organized as follows. In Section 2, the concepts of morphological filter and directional morphology are presented. In Section 3 the notions of line-segment and orientation functions, derived from the supremum of directional erosions, are introduced. Also in the this section, an algorithm to compute the line-segment and the orientation function is analyzed. Next, in Section 4 an approach of working with directional morphology, the watershed transform and a region adjacency graph (RAG) for segmenting directional structures is proposed.

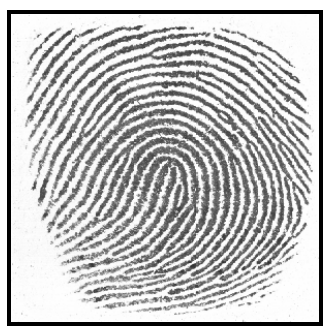

(a)

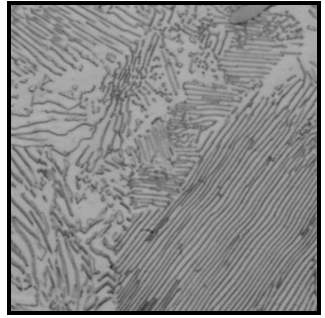

(b)

Fig. 1. (a) Fingerprint image, (b) Pearlitic phase image

\section{Basic Morphological Directional Transformations}

Mathematical morphology is based principally on so-called increasing transformations [141516. Between the different morphological tools, an interesting class of transformations are the directional ones. Morphological directional transformations are characterized by two parameters. A structuring element L depends on its length $(\operatorname{size} \mu)$ and on the slope (angle $\alpha$ ) of the element. Thus, the set of 
points of a line segment $L(\alpha, \mu)$ is computed by two sets of points for $\alpha \in[0,90]$. The sets of points $\left\{\left(x_{i}, y_{i}\right)\right\}$ defined by the following expressions:

$$
\begin{aligned}
& \text { if } 0 \leq \alpha \leq 45 \text { then, } y_{i}=x_{i} \tan (\alpha) \text { for } \quad x_{i}=0,1, \cdots, \mu \cos (\alpha) \\
& \text { if } 90 \geq \alpha>45 \text { then, } x_{i}=y_{i} \cot (\alpha) \text { for } \quad y_{i}=0,1, \cdots, \mu \cos (\alpha)
\end{aligned}
$$

and the set of points $\left\{\left(-x_{i},-y_{i}\right)\right\}$. This means, the structuring element is a symmetric set $L(\alpha, \mu)=\hat{L}(\alpha, \mu)$. Similar expressions can be described for $\alpha \in$ $(90,180]$. Then, the morphological opening and closing are given by:

$$
\gamma_{L(\alpha, \mu)}(f)=\delta_{L(\alpha, \mu)}\left(\varepsilon_{L(\alpha, \mu)}(f)\right) \quad \text { and } \quad \varphi_{L(\alpha, \mu)}(f)=\varepsilon_{L(\alpha, \mu)}\left(\delta_{L(\alpha, \mu)}(f)\right)
$$

where the morphological erosion and dilation are given by: $\varepsilon_{L(\alpha, \mu)}(f)(x)=$ $\wedge\{f(y): y \in L(\alpha, \mu)(x)\}$ and $\delta_{L(\alpha, \mu)}(f)(x)=\vee\{f(y): y \in L(\alpha, \mu)(x)\}$.

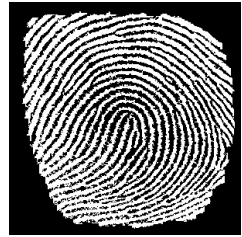

(a)

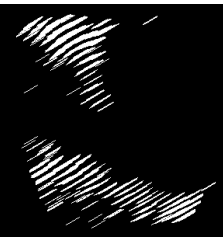

(b)

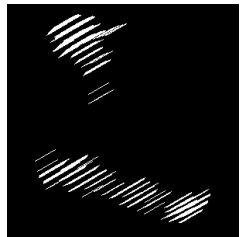

(c)

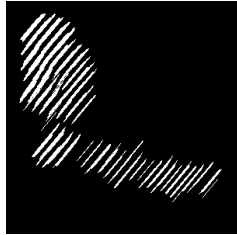

(d)

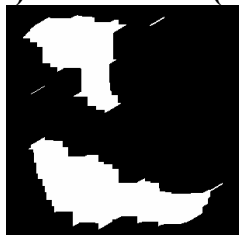

(e)

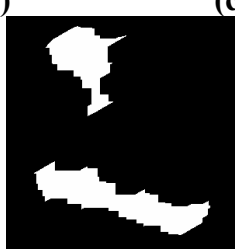

(f)

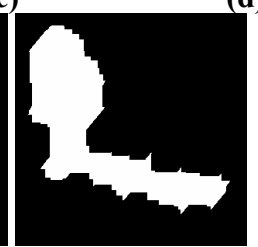

(g)

Fig. 2. Original image, (b), (c) Directional openings at direction 30 degrees with size 20 and 30, respectively, (d) Directional opening at direction 50 degrees size 30, (e), (f) and (g) Morphological closings size 10 of images in 2 (b), (c) and (d), respectively

\section{Size and Orientation Codification Based on Directional Erosions (Line-Segment and Orientation Functions)}

\subsection{Line-Segment and Orientation Functions}

In this section and in the following ones we will look for an approach to detect directional structures where the connectivity notion plays a fundamental role for segmenting them. In fact, it is well-known that the notion of connectivity is linked to the intuitive idea of segmentation task, where the objective is to split the connected components into a set of elementary shapes that will be processed 
separately. Then, the problem lies in determining what a connected component is for an image such as those illustrated in Fig. 1. One can take different ways for introducing such a concept. For example, in Fig. 2 some orientation structures are extracted using directional openings. Then, the directional structures are determined by a clustering process computed in this case by a morphological closing. Figures 2 (b) and (c) show the directional openings at direction 30 degrees with sizes of the structuring elements of 20 and 30, respectively. A hierarchy of structure sizes can be introduced by means of the granulometry. Thus, the directional granulometry and a connectivity introduced by means of closings for extracting some clustering enable us to compute the directional structures as illustrated in Figs. 2 (e) and (f). Nevertheless, the computing of the directional structures by this approach can become very complex. For instance, in Figs. 2 (d) and (g) the directional structures were determined for an angle of 50 degrees and a scale of 30. Some regions of the structures of the images in Figs. 2 (c) and (f) are the same than those of the images in Figs. 2 (d) and (g) (i.e., the intersection between these images is not empty). Given that deficiency, we look for another approach where the information of scales and directions of the structures of the image are easily accessible. Two functions that codify the size and the orientation are introduced below. The idea for codifying size structure comes from the notion of the distance function. Remember that the distance function $D_{X}(x)$ is a transformation that associates with each pixel x of a set $\mathrm{X}$ its distance from the background. Let us now to define a new function derived from the notion of distance function.

Definition 1. The line-segment function $D m_{X}(x)$ is a transformation that associates with each pixel $x$ of a set $X$ the length of the longest symmetrical line segment, centered at the origin, placed at point $x$ and completely included in $X$.

The goal of building this function consists in codifying the size information in such a way that local directional information can be accessed from each point of the function. This codification of the size information will be used to build a local approach for detecting directional structures on an image. This function, which we call line-segment function $D m_{X}$, is computed by using the supremum of directional erosions. To stock the size information for all $\lambda$ values, a gray-level image $D m_{X}$ is used. Let $\mathrm{X}$ be a given set, one begins with a small structuring element by taking into account all orientations to compute the set $\operatorname{Sup}_{\alpha \in[0,180]}\left\{\varepsilon_{L(\lambda, \alpha)}(X)\right\}$. That means, one takes all points of the image that are not removed by at least one of the directional erosions. Then one increases $D m_{X}$ by one at all points x belonging to the set $S u p_{\alpha \in[0,180]}\left\{\varepsilon_{L(\lambda, \alpha)}(X)\right\}$, and one continues the procedure by increasing the size of the structuring element until the structure (the image) is completely removed. This means that the procedure continues until one has a $\lambda_{\max }$ value such that $\operatorname{Sup}_{\alpha \in[0,180]}\left\{\varepsilon_{L\left(\lambda_{\max }, \alpha\right)}(X)\right\}=\emptyset$. Figures 3 (b) and (c) show the output images computed from the original image in Fig. 3) (a) for the size values 40 and 60, respectively. As expressed before, the gray-levels of the function $D m_{X}$, are the sizes of the longest lines that can be included in the structure. Whereas, for the structures that can be considered as composed as a set of lines, as those in Figs. 1(a) and (b), we assume that 
the the maxima of the function $D m_{X}$ play a main role since they codifies the longest lines that take the whole context of the image. Thus, one knows the position of the largest structuring elements that can be included completely in the structure. However, the angles of these structuring elements (line segments) are not accessible from the image $D m_{X}$. Then, let us introduce a second function associated to the line-segment function.

Definition 2. The orientation function $O m_{X}(x)$ is a transformation that associates with each pixel $x$ of a set $X$ the angle of the longest symmetrical line segment, centered at the origin, placed at point $x$ and completely included in $X$.

Therefore, one stocks the directions of the line segments in a second image $O m_{X}$, called orientation function, when the line-segment function is computed. A real example (pearlitic phase micrograph) is shown in Fig. 4. The images in Fig. 4(b)-(c) illustrate the line-segment function $D m_{X}$ image and the image containing the orientation $\mathrm{Om}_{X}$, respectively, computed from the binary image in Fig. 4(a). These functions can now be used for computing the line segments that characterize the structure. The line-segment function and its associated orientation image containing the angles serve to suggest a method for segmenting images with directional structures.

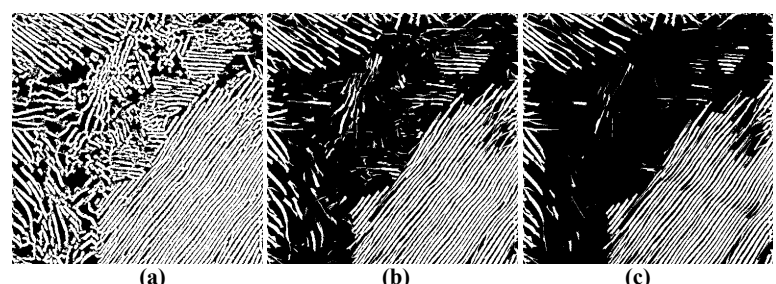

Fig. 3. (a)Original image, (b) and (c) $\operatorname{Sup}_{\alpha \in[0,180]}\left\{\varepsilon_{L(\lambda, \alpha)}(X)\right\}$ with $\lambda 40$ and 60

\subsection{Non-parametric Algorithm to Build the Line-Segment and Orientation Functions and the Limits of the Approach}

Since the number of operations for computing the line-segment function and the orientation function is considerable, one can suppose that the method is very expensive in computation time. Moreover, the building of these functions seems to require a size parameter (the largest structuring element) and an angle step to compute them. Let us illustrate an algorithm to build these functions that does not require any parameter and that is not expensive in computation time. First, concerning the size parameter (largest structuring element), it was fixed to the size of the image diagonal that is the size of the largest structure that can be include in the image. Let $S_{x}$ and $S_{y}$ be the dimension of the images, horizontal and vertical axis, respectively. For example, for a VGA image $640 \times 480$, one has $L=\sqrt{S_{x}^{2}+S_{y}^{2}}=800$, then the largest structuring element has a size of 


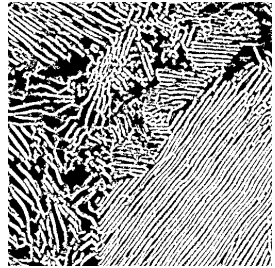

(a)

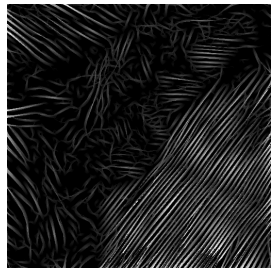

(b)

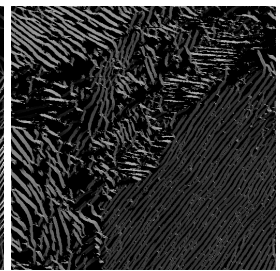

(c)

Fig. 4. (a) Original Binary image, (b) Line-segment function, (c) Orientation function

400 since one uses symmetrical structuring elements. It is clear that few cases of images, containing such structure characteristics, can be found in real images. Next, one requires to fixe the step in degrees to compute the line segments. In practice, a step between 5 and 10 degrees is sufficiently, but let us fix it to a smaller value (one degree) in order to show the limiting case in computation time. Then, one computes 180 structuring elements of size $L / \mathcal{L}$, and they are stocked in a structure data (list of lists). Since symmetrical structuring elements are used, only half of the straight lines is stocked and centered at origin $(0,0)$. In fact, only the Freeman codes are stocked. Let $\left\{L s_{i}\right\}$ with $i \in\{0,1,2, \ldots, 179\}$ be the lists containing the Freeman codes required to build a half of the structuring elements and let $L s_{j}$ be a given list. The structuring element is built using the list $L s_{j}=\left\{c_{k}\right\}$ with $c_{k} \in\{0,1,2,3, \ldots, 7\}$ and its symmetrical data $\check{L} s_{j}=\left\{\check{c}_{k}\right\}$ with $\check{c}_{k}=\left(c_{k}+4\right) \bmod 8$. Consider the example in Fig. 5(a) where an erosion by a line segment is applied to the structure in gray color. The structuring element is obtained from the list $L s_{j}=\{0,1,0,1,0,1,0,1,0\}$ (blue 0 and red 1) and $\check{L} s_{j}=\{4,5,4,5,4,5,4,5,4\}$ (green 4 and yellow 5 ). Now, to compute the erosion at point $(\mathrm{x}, \mathrm{y})$ of an image $f$ marked by a white dot, one begins by computing the smallest value between the points $(\mathrm{x}-1, \mathrm{y}),(\mathrm{x}, \mathrm{y})$ and $(\mathrm{x}+1, \mathrm{y})$. Then, the erosion size one is given by the infimum (the intersection for sets) $\varepsilon_{1}(f)(x, y)=f(x-1, y) \bigwedge f(x, y) \bigwedge f(x+1, y)$. Next, one computes the erosion size 2 with the following two points of the structuring element $(\mathrm{x}-2, \mathrm{y}+1)$ and $(\mathrm{x}+2, \mathrm{y}+1)$ and the erosion size one $\varepsilon_{1}(f)(x, y)$, thus, $\varepsilon_{2}(f)(x, y)=f(x-2, y-$ 1) $\bigwedge \varepsilon_{1}(f)(x, y) \wedge f(x+2, y+1)$. The procedure continues until the last pair of points of the structuring element is taken into account. In this example, one requires a longer structuring element to remove the point $(x, y)$ of the image. Nevertheless, in the example in Fig. $5(\mathrm{~b})$, when the third erosion is applied, the point is removed; i.e., $\varepsilon_{3}(f)(x, y)=f(x-3, y-2) \wedge \varepsilon_{2}(f)(x, y) \wedge f(x+3, y+3)=$ 0 , then, the procedure is stopped. This procedure is applied at each point of the image. It is clear that the fact of using the infimum (AND operation in a computer) to compute the erosion and to stop the procedure when it is no longer required, permits one to compute the erosion of the image faster. Then, instead of calculating the $\operatorname{Sup}_{\alpha \in[0,180]}\left\{\varepsilon_{L(\lambda, \alpha)}\right\}$, one computes at each point $x$ of the image, the longest structuring element that can not remove this point. Next, the length of this structuring element is used to affect the function $D m_{X}$ at point $x$. For instance, in the example in Fig. 1 (b), an image of size $512 \times 512$ pixels, 5 
seconds are required to compute the line-segment and orientation images using an angle step of one degree, whereas working with a step of 5 degrees in the interval $[0,180]$ a second is only required. For the image in Fig. 11(a) (300x300 pixels) one requires less than two seconds using an angle step of one degree. The computer, that has been used for the experiments, is a laptop with $1.59 \mathrm{Ghz}$ processor and $256 \mathrm{MB}$ in RAM.

\subsection{The Limits of the Approach}

Let us take some geometrical examples to illustrate some limitations of the approach above proposed to extract the directional characteristics. The first example is the case of a rectangle of length $l$ and wide $h$ as shown in Fig 5 (c) and (d). The longest line segments that can be placed inside the rectangle are the diagonal lines of length $\left[l^{2}+h^{2}\right]^{1 / 2}$ and their angle is given by $\alpha=\tan ^{-1}(h / l)$. It is clear that larger is $l$ and the smaller is $h$, then closer to 0 degrees is $\alpha(l \rightarrow \infty$ or $h \rightarrow 0, \Rightarrow \alpha \rightarrow 0)$. Consider an example where the rectangle has $l=100$ pixels. Then, for $h=20$, one has $\alpha=11.5$ degrees, for $h=10, \alpha=5.7$ degrees, for $h=5, \alpha=2.8$ degrees. Another source of errors are the rectangle extremes, since at the limits the angle of the longest line (in this example) has an angle valu of 90 degrees. Moreover, the points remaining in the line that cuts the rectangle along the horizontal at $h / 2$ crossing its center, can change between $\alpha=\tan ^{-1}(h / l)$ and 90 degrees. Figures 5 (c) and (d) show two examples in color representation. This drawback could be avoided by applying the supremum of directional openings, however, the computation time in determining the longest lines included in the structure will increase considerably. To illustrate that, remember the traditional geometrical interpretations of the erosion and the opening. In the erosion case one selects the center of the structuring element completely include in the structure, whereas in the opening case all the points hitting the structuring element are chosen. This means, when an opening is applied, the points of the image are analyzed several times and it will not possible to apply the algorithm above proposed. Now let us analyze another geometrical example given by a shape formed by two concentric circles of different radii (annulus or ring of wide $\mathrm{h}$ ) as illustrated in Fig. 5 (e). In the case of a disk, the maximum symmetrical distance remains at the middle point of the segment joining any two points of the circle (chord), the longest chord going through the center of the disk (diameter of the disk). In the case of a ring the longest line segment is tangent of the inner circle, and the smallest line is zero, a point on the outer circle. Between these two extremes there exit different chords all at the same angle. Then, when working with structures with a given curvature the error of the direction is smaller than the case of a straight right (or without error when the region of a shape is assimilable to a ring) as illustrated in Fig. [5] (f). However, the maxima of the line-segment function will remain close to the contour (inner contour) of the structure. The limits of the approach, shown in this section, are attenuated when the image is composed by thin structures as those in Fig. 1 (b) or when the structures have a curvature as those in Fig. 1(a). To better illustrate the performance of the line-segment and the orientation functions several examples 
are shown in Fig. 6. Three examples of orientation detection in fingerprints are carried out. To illustrate the orientation detection the line segment and orientation functions were computed from the binary images obtained from the original images illustrated in Figs [6 (a), (b), and (c), then a line segment was placed at some maxima of the line segment function by taking into account the direction given by the orientation function as illustrated in Figs. 6 (d), (e) and (f). Only one point of each regional maximum was selected to place a line segment.

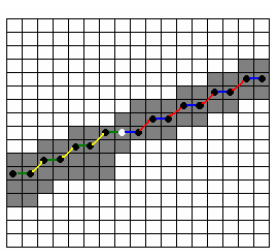

(a)

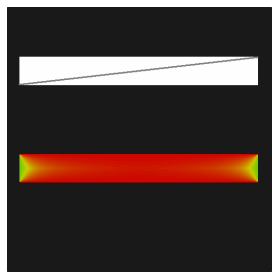

(d)

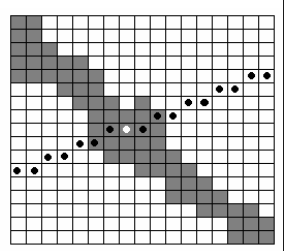

(b)

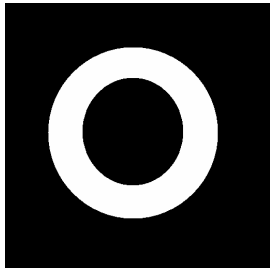

(e)

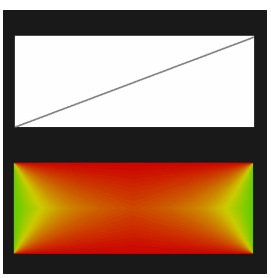

(c)

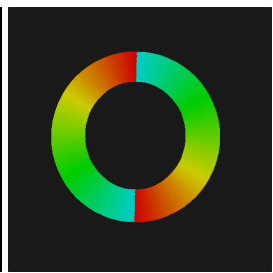

(f)

Fig. 5. a) and b) Directional erosions, c) and d) approach limits in rectangle structures, e) and f) approach limits in disk structures

\section{Image Segmentation Using Directional Morphology and the Watershed Transformation}

Image segmentation is one of the most interesting problems in image processing and analysis. The main goal in image segmentation consists in extracting the regions of greatest interest in the image [17/1819]. In mathematical morphology, the watershed-plus-marker approach is the traditional image segmentation method [17. Here, an alternative approach for segmenting images with directional structures is applied. Instead of looking for a set of markers signaling the regions, the watershed will be applied directly to obtain a fine partition. Then a systematic merging process will be applied to obtain the final segmentation. However, to carry out the merging process it is preferable to work with the catchment basins associated with the watershed image. Figure 7(a) shows the catchment basins, computed from the inverse line-segment function shows in Fig. 4(b) and weighted by the values of the angles of of the orientation function $O m_{X}$ in Fig. 4(c). In order to take into account the neighborhood relationships a region 


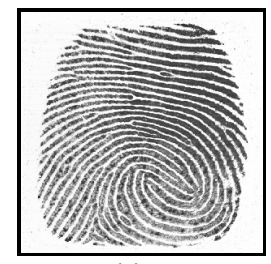

(a)

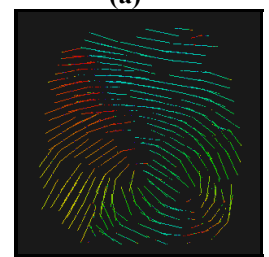

(d)

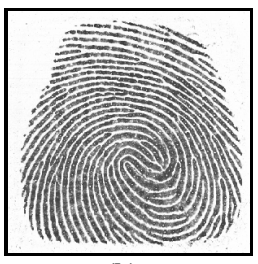

(b)

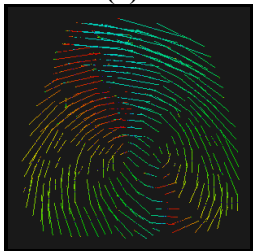

(e)

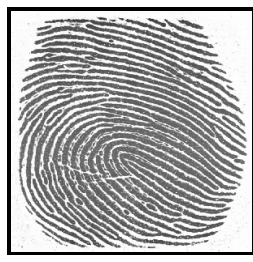

(c)

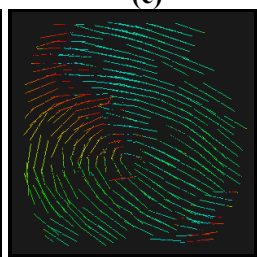

(f)

Fig. 6. a), b) and c) Original images, d), e) and f) Main orientations of images (a), (b) and (c), respectively

adjacency graph (RAG) must be computed. The RAG graph is constructed by using of the catchment basins of the image in Fig Z7(a). One takes a point from each minimum of the inverse line-segment function for representing each catchment basin. The neighborhood graph of the maxima of the line-segment function $D m_{X}$ and the orientation $O m_{X}$ function synthesize the directional structures of the image. Once the regions are codified on a graph, we can compute the directional structures based on the valued graph. The following method (see [20]) for reducing the numbers of regions may be carried out: a) Each border has assigned an orientation distance between the two regions it separates, b) The borders are sorted in increasing order, c) Two regions separated by the smallest distance are merged, d) The step (b) is repeated until the criterion can not be satisfied. We illustrate the method by identifying the adjacent regions with more-or-less similar orientation by considering the image in Fig. 1(b), a micrograph of the pearlite structure in steel. To achieve such a goal, one merges the vertices (catchment basins) with a difference of angles smaller than or equal to a given angle value $d\left(R_{i}, R_{j}\right)=\left|\operatorname{angle}\left(R_{i}\right)-\operatorname{angle}\left(R_{j}\right)\right| \leq \theta$. Figure 7 (c) shows in color levels the orientation function of Fig. 4 (c), while Fig. 7 (d) shows the output image after the merging process using angle difference criteria $\theta$ of 20 . The intensities of regions shown in Figs. 7 (b) and (d) were taken to be proportional to the mean value of the merged region angles. Once the grains of the perlitic structure are separated, it is now possible to compute some measures (for example, a granulometric study). It is clear that, when the regions of the image are codified under the form of a graph, many criteria can be easily introduced to segment the image. Figure 7 (e) shows this advantage of using a RAG structure for the merging process since the introduction of other criteria can improve the final segmentation. However, in this example, instead of computing the catchment basins on the whole image, one can do better by computing the weighted partition in a geodesic way. The mask, where the catchment basins transform will be 


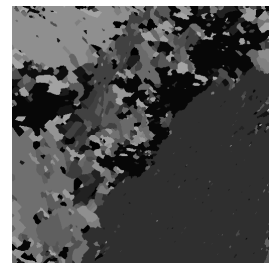

(a)

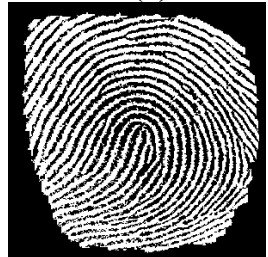

(e)

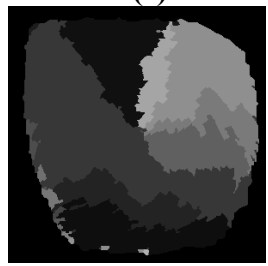

(i)

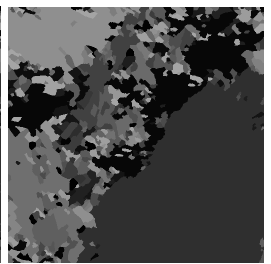

(b)

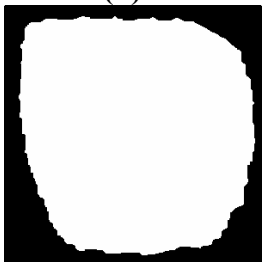

(f)

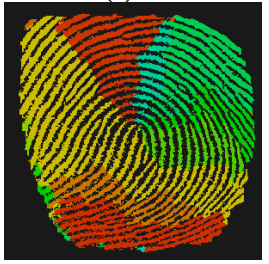

(j)

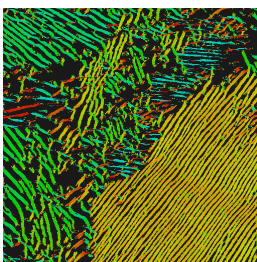

(c)

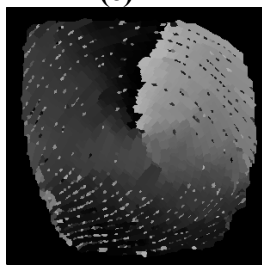

(g)

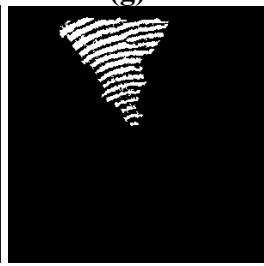

(k)

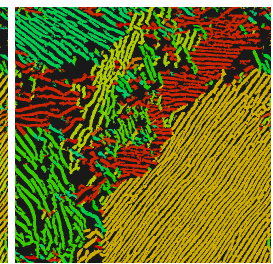

(d)

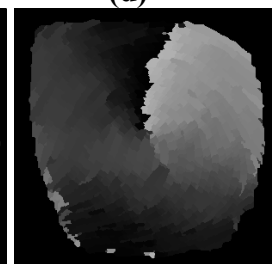

(h)

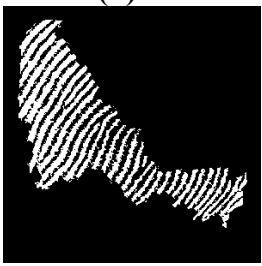

(l)

Fig. 7. a) Catchment basins of the partition weighted by the orientation (angles), b) Merged regions, c) and d) Orientation function and segmented image in color representation, e) Original binary image, f) Mask image, g) Catchment basins of the partition weighted by the orientation, h) Filtered partition by size criterion, i) Segmented partition, j) Color representation, k) and i) connected components

applied, is given by the image in Fig. 7 (f). This last image is the output image obtained by the morphological closing size 6 of the original image in Fig. 7 (e). Then, the image in Fig. $7(\mathrm{~g})$ is computed by the catchment basins transform inside the mask. Since thin connections exist between the directional structures of the directional structures, one observes in Fig. 7 (g) small regions that are not representative of the structure (from a segmentation point of view). A size criterion was introduced to remove these regions as illustrated in Fig. 77(h). Once the small regions are removed, two other criteria can be applied for obtaining the final segmentation. Let $\vec{\mu}_{i}$ and $\vec{\sigma}_{i}$ be the mean and the variance values in the region $R_{i}$. Then, two regions $R_{i}$ and $R_{j}$ can be merged if the orientation distance $d_{\vec{\mu}}\left(R_{i}, R_{j}\right)=|\vec{\mu}(i)-\vec{\mu}(j)| \leq \theta$. This means, after the merging process between regions is carried out, the mean value is used to describe the new region and a new variance value is computed. However, even if the mean difference criterion is satisfied, but one of the regions has a great variance $\left(\vec{\sigma}_{i}>\tau\right.$ or $\vec{\sigma}_{j}>\tau$ ), these regions will not be merged. Figures 7 (i) and (j) show the segmented images, in gray and color levels, using a mean orientation difference criterion $\theta=15$ degrees and using a variance criterion $\tau=6.5$. In Section 3, 
we commented that the notion of connectivity is linked to the intuitive idea of segmentation. Under our approach the images in Figs. $7(\mathrm{k})$ and (l) show two connected components of the original image in Fig.7. Compare these connected components with those of Figs. 2 (e), (f) and (g). From the point of view of fingerprint recognition, the connected components illustrate the existence of a singular point (core). The largest component in Fig. 7(1) describes a separation with the topmost curving that enables to classify this fingerprint. The image in Fig. 7 (i) shows clearly the existence of the core.

\section{Conclusion}

This paper has shown the possibilities for application of morphological directional transformations to segment images with directional structures. One proposes a local approach that involves a local analysis using the concepts of the line-segment and orientation functions proposed in this paper. The maxima of the line-segment function were used for computing the loci of maximal structuring elements, and the orientation function was used to obtain the angles of the line segments. These pairs of local parameters enable us to produce a good description of the image by means of line segments. Then, a partition of the image may be computed by means of the catchment basins associated with the watershed transform. This enables us to realize a neighborhood analysis, using a RAG structure, in order to merge adjacent regions of the partition according to appropriate criteria, thus segmenting the images into connected components. The results based on the algorithms presented in this paper show the good performance of the approach.

Acknowledgements. The author Luis Morales acknowledges the government agency CONACyT for the financial support. The author I. Terol would like to thank Diego Rodrigo and Darío T.G. for their great encouragement. This work was funded by the government agency CONACyT, Mexico.

\section{References}

1. Soille, P., Talbot, H.: Directional morphological filtering. Trans. on Pattern Anal. Machine Intell. 23(11), 1313-1329 (2001)

2. Soille, P., Breen, E.J., Jones, R.: Recursive implementation of erosions and dilations along discrete lines at arbitrary angles. IEEE Trans. on Pattern Anal. Machine Intell. 18(5), 562-567 (1996)

3. Jeulin, D., Kurdy, M.: Directional mathematical morphology for oriented image restoration and segmentation. Acta Stereologica 11, 545-550 (1992)

4. Tuzikov, A., Soille, P., Jeulin, D., Vermeulen, P.: Extraction of grid patterns on stamped metal sheets using mathematical morphology. In: Proc. of International Conference on Pattern Recognition, vol. 1, pp. 425-428 (1992)

5. Oliveira, M.A., Leite, N.J.: Reconnection of fingerprint ridges based on morphological operators and multiscale directional information. In: Proc. of XVII Brazilian Symposium on Computer Graphics and Image Processing, pp. 122-129 (2004) 
6. Morales-Hernández, L.A., Terol-Villalobos, I.R., Dominguez-González, A., HerreraRuiz, G.: Characterization of fingerprints using two new directional morphological approaches. In: Advances in Dynamics, Instrumentation and Control, pp. 325-334. World Scientific Publishing Co, Singapore (2007)

7. Cappelli, R., Lumini, A.: Fingerprint classification by directional image partitioning. IEEE Trans. on Pattern Anal. Machine Intell. 21(5), 402-421 (1999)

8. Park, C.H., Lee, J.J., Smith, M.J.T., Park, K.H.: Singular point detection by shape analysis of directional fields in fingerprints. Pattern Recognition 39, 839-855 (2006)

9. Li, J., Yau, W.Y., Wang, H.: Constrained nonlinear models of fingerprint orientations with prediction. Pattern Recognition 39, 102-114 (2006)

10. Lee, J.K., Newman, T.S., Gary, G.A.: Oriented connectivity-based method for segmenting solar loops. Pattern Recognition 39, 246-259 (2006)

11. Bahlmann, C.: Directional features in online handwriting recognition. Pattern Recognition 39, 115-125 (2006)

12. Kass, M., Witkin, A.: Analyzing oriented pattern. Computer Vision, Graphics, and Image Processing 37(3), 362-385 (1987)

13. Bazen, A.M., Gerez, S.H.: Systematic methods for the computation of the directional fields and singular points of fingerprints. IEEE-Trans. on Pattern Analysis and Machine Intelligence 24(7), 905-919 (2002)

14. Serra, J.: Image Analysis and Mathematical Morphology. Theoretical advances, vol. II. Academic Press, London (1988)

15. Heijmans, H.J.A.M.: Morphological Image Operators. Academic Press, New York (1994)

16. Soille, P.: Morphological Image Analysis: Principles and Applications, 2nd edn. Springer, Heidelberg, Berlin (2003)

17. Meyer, F., Beucher, S.: Morphological segmentation. J. Vis. Comm. Image Represent 1, 21-46 (1990)

18. Crespo, J., Schafer, R., Serra, J., Meyer, F., Gratin, C.: A flat zone approach: A general low-level region merging segmentation method. Signal Process 62, 37-60 (1997)

19. Salembier, Ph., Serra, J.: Morphological multiscale image segmentation. In: Proc. SPIE-Visual Communications and Image Processing, vol. 1818, pp. 620-631 (1882)

20. Shafarenko, L., Petrou, M., Kittler, J.: Automatic watershed segmentation of randomly textured color images. IEEE Trans. on Image Processing 6(11), 1530-1544 (1997) 